\title{
Synthesis of B-Sb by rapid thermal annealing of B/Sb multilayer films
}

\author{
S N DAS and A K PAL* \\ Department of Instrumentation Science, Jadavpur University, Kolkata 700 032, India
}

\begin{abstract}
Group III-V compound B-Sb films were synthesized from B/Sb/.../B multilayer films deposited by electron gun evaporation onto silicon substrate and subjecting the above multilayer to rapid thermal annealing at $773 \mathrm{~K}$ for 3 min. The films were characterized by XRD, TEM, XPS and optical studies. XPS studies indicated the ratio of $B: S b \sim 1$. XRD and electron diffraction patterns indicated the reflections from (100), (111), (102) and (112) planes of zinc blende BSb. Band gap evaluated from optical studies was $\sim 0.51 \mathrm{eV}$. Refractive index of the films varied between 1.65 and 2.18 with increasing energy of incident photon and plasma frequency $\left(\omega_{\mathrm{p}}\right)$ was estimated to be $\sim 2.378 \times 10^{-14} \mathrm{~s}^{-1}$. The effective mass was computed to be $\sim 0.0845 \mathrm{~m}_{\mathrm{e}}$.
\end{abstract}

Keywords. III-V Compound; optical property; XPS.

\section{Introduction}

Boron compounds have a number of remarkable properties that distinguishes them from other III-V compounds. The unusual properties have the origin from the small core size and the absence of $p$ electrons in boron (Ferhat $e t$ al 1998). The interesting results concerning the electronic properties of this compound has culminated in developing right kind of synthesis technique for BSb films. There are not very many studies on the synthesis of this material due to the inherent difficulty involved in the synthesis. Recently, Kumashiro et al (2002) reported the synthesis of BSb films by molecular flow region PVD method using decaborane powder as boron source and evaporated antimony as antimony source. They also reported the measurement of electrical and thermoelectric properties on the same film (Kamashiro et al 2004).

In this paper, we report the synthesis of BSb films by evaporating $\mathrm{B}$ and $\mathrm{Sb}$ by e-gun to form $\mathrm{B} / \mathrm{Sb}$... Sb/B multilayer with predetermined thickness of boron and antimony and subsequently subjecting the multilayer to rapid thermal annealing. The films were characterized by measuring microstructural, optical and compositional properties.

\section{Experimental}

Multilayer films of B and Sb were deposited onto Si and fused silica substrates at room temperature by e-gun evaporation of boron (99.99\%) and antimony (99.995\%). Total thickness of the stack thus prepared was $\sim 22 \mathrm{~nm}$. The above stack was subjected to rapid thermal annealing at $773 \mathrm{~K}$ for $3 \mathrm{~min}$. The resultant films were characterized by measuring optical, microstructural and compositional properties. Optical studies were performed by measuring

\footnotetext{
*Author for correspondence (msakp2002@yahoo.co.in)
}

transmittance in the wavelength region $\lambda=500-2400 \mathrm{~nm}$ (resolution of $\lambda, \sim 0.07 \mathrm{~nm}$ ) using a spectrophotometer at room temperature. The films deposited directly on carbon coated copper grids were used for TEM studies.

The XPS spectra were recorded using a VG Microtech XPS set up incorporated with $1256.4 \mathrm{eV} \mathrm{MgK}_{\alpha} \mathrm{X}$-ray source. The films were also characterized by X-ray diffraction (XRD) studies using $\mathrm{Cu} \mathrm{K} \alpha$ line $(0 \cdot 154 \mathrm{~nm})$.

\section{Results and discussion}

\subsection{Microstructural studies}

It may be observed that the as-deposited multilayer film indicated (figure 1a) peaks for elemental boron and antimony only. With the increase in temperature during RTA, the individual peaks for $\mathrm{B}$ and $\mathrm{Sb}$ smoothened out and when annealed at temperature, $\sim 773 \mathrm{~K}$, for 3 min culminated in possible complete synthesis of BSb film as indicated by the presence of characteristic peaks for BSb only (figure 1b). The peaks that matched well with the calculated lattice spacing $(d)$ values for different planes appeared at $2 \theta=23.78^{\circ}$ for reflections from (101) planes of BSb. Two peaks corresponding to reflections from (220) and (330) planes for zinc blend-BSb (ZB-BSb) could also be observed at $2 \theta=48.48^{\circ}$ and $76 \cdot 33^{\circ}$, respectively. It may be noted here that the peak for reflection (300) planes for Sb which appeared for the as-deposited multilayer films (figure 1a) gradually disappeared with increasing temperature, $\sim 673 \mathrm{~K}$, during annealing. TEM micrograph and corresponding electron diffraction pattern of two representative films with nearly equal B : Sb ratio are shown in figure 2 . Both the micrographs indicated well-dispersed grains and the diffraction pattern showed characteristic spots for planes (111), (102) and (112) in figure 2a. The diffraction pattern shown in figure $2 \mathrm{~b}$ indicated two additional spots for reflec- 


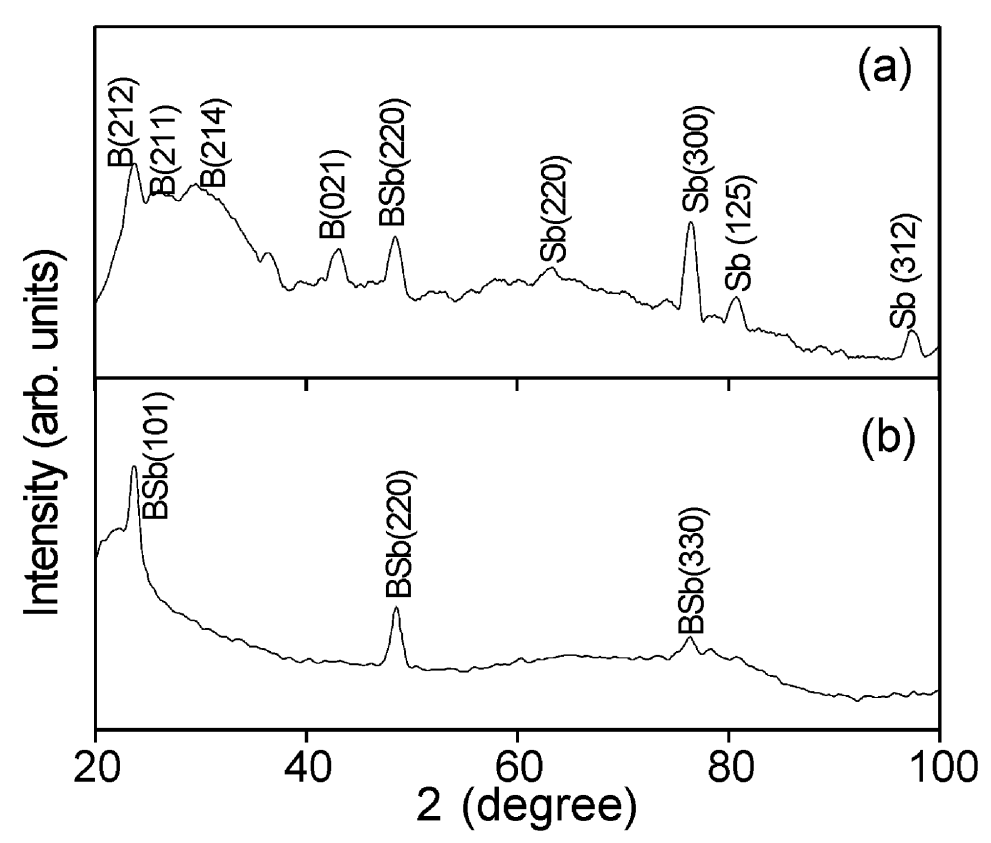

Figure 1. XRD spectra for two representative films: (a) as-deposited and (b) after RTA at $773 \mathrm{~K}$ for $3 \mathrm{~min}$.
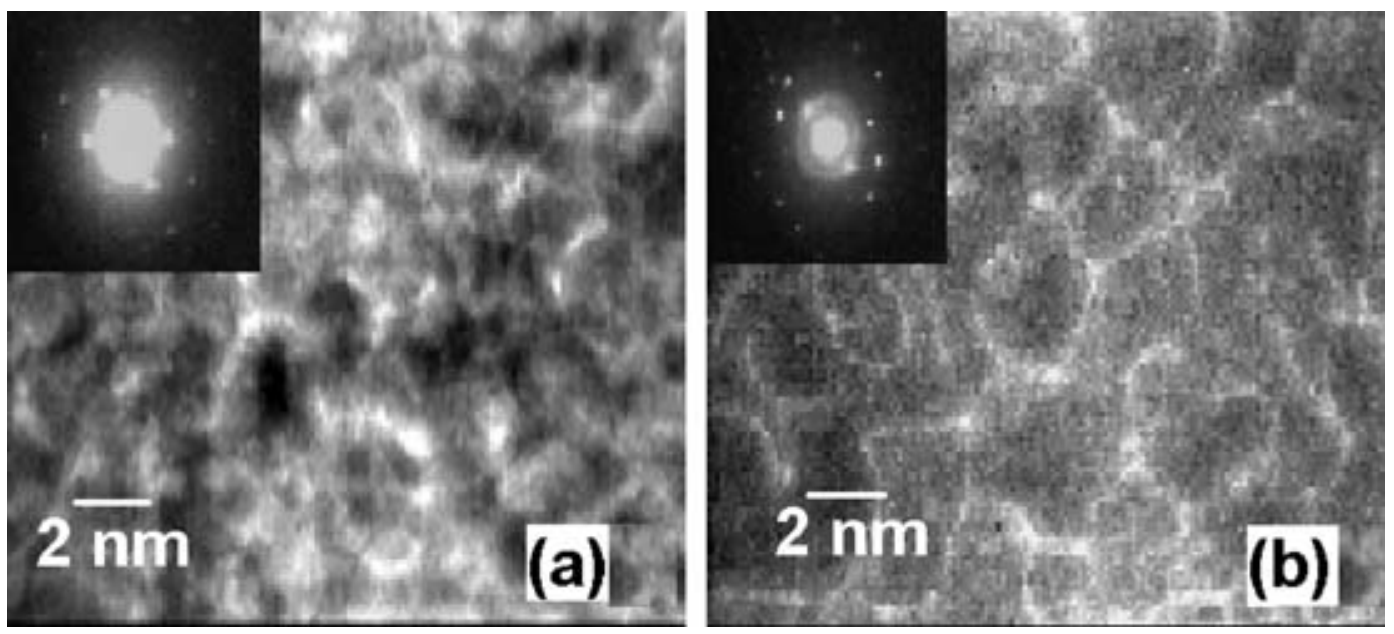

Figure 2. TEM micrographs of two representative BSb films after RTA.

tion from (220) and (330) planes for ZB-BSb. Presence of twin planes could be appreciated from this diffraction pattern. The structural and microstructural properties as obtained from XRD and TEM studies, respectively indicate the synthesis of BSb films by this technique. HRTEM images are shown in figure 3 . The $d$ spacing obtained for the films are $\sim 0.301 \mathrm{~nm}$ and $0 \cdot 212 \mathrm{~nm}$ and matches well for planes (111) and (112) of ZB-BSb.

\subsection{XPS study}

Photoelectron spectroscopy (XPS) would supplement confirmative information regarding the formation of BSb. Figures 4(a) and (b) show a typical XPS spectrum for a representative film. This study confirms the presence of boron and antimony. Besides the peaks for $\mathrm{B}$ and $\mathrm{Sb}$, two additional peaks with low intensity were noticed for $\mathrm{C}$ and $\mathrm{O}$ in the XPS spectra. The presence of traces of $\mathrm{C}$ is due to the vacuum level $\left(\sim 10^{-6}\right.$ Torr $)$ in the chamber while the peak for $\mathrm{O}$ could be due to oxygen during handling the film outside the chamber. XPS spectra for B $(1 s), \mathrm{Sb}-3 d_{5 / 2}$ and $\mathrm{Sb}-3 d_{3 / 2}$ are shown in figure 4 . Thus, one would expect that the peak position of $\mathrm{B}(1 s)$ for BSb films may result in shift in peak position. It may be observed from figure 4 a that $\mathrm{B}(1 s)$ peak has a shoulder at $\sim 195 \mathrm{eV}$. This peak could be deconvoluted showing the existence of two peaks at $\sim 191.7 \mathrm{eV}$ and $195.2 \mathrm{eV}$. The peak at $191.7 \mathrm{eV}$ could be 

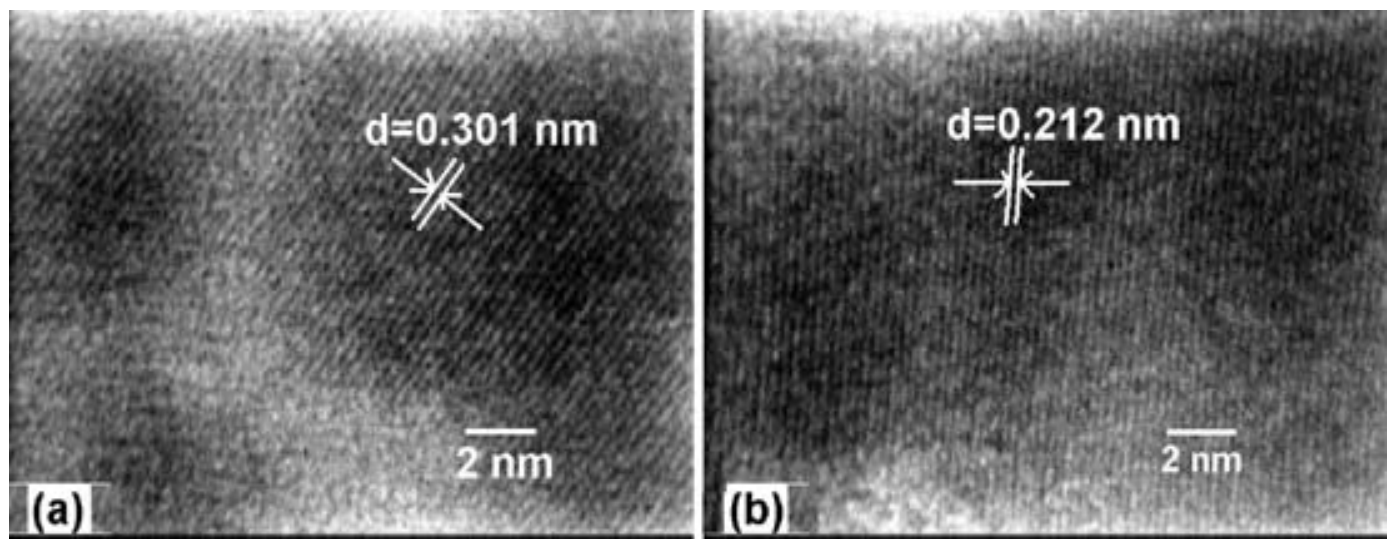

Figure 3. HRTEM picture of two representative films whose micrographs are shown in figure 2.
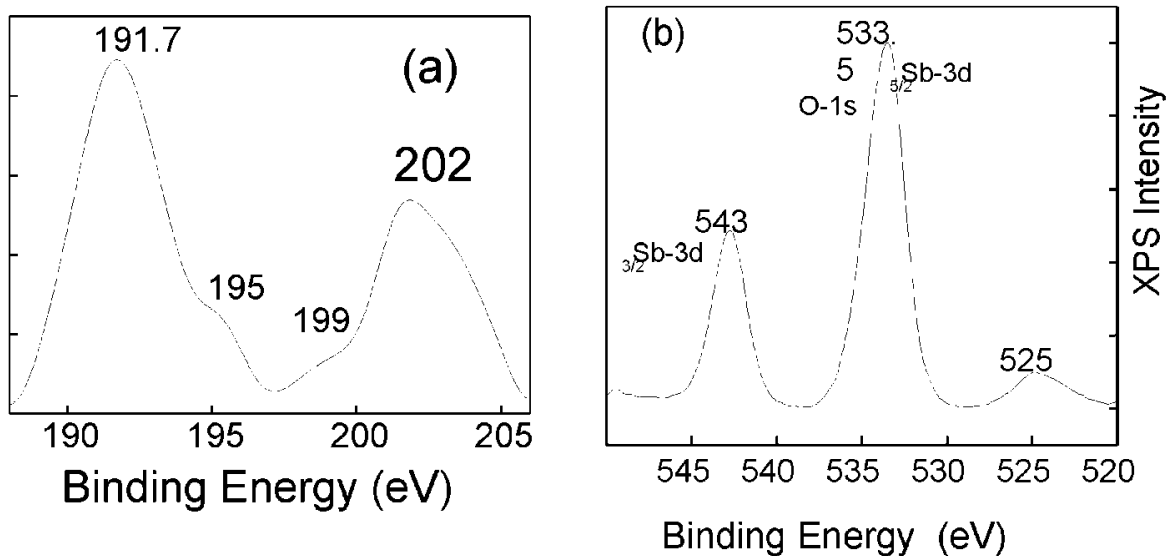

Figure 4. XPS spectra for (a) boron and (b) antimony.

ascribed to B in BSb. The shoulder observed at $\sim 195 \mathrm{eV}$ for the peak for $\mathrm{B}(1 s)$ could be associated with $\mathrm{B}-\mathrm{O}$ bond due to the presence of physisorbed oxygen at the surface. The observed XPS spectra for Sb consist of two peaks for $\mathrm{Sb}-3 d_{5 / 2}$ and $\mathrm{Sb}-3 d_{3 / 2}$. The peak for $\mathrm{Sb}-3 d_{5 / 2}$ may overlap with the peak for $\mathrm{O}(1 s)$ and deconvolution of the same generated two peaks for Sb-3 $d_{5 / 2}$ and $\mathrm{O}(1 s)$ as shown in figure $4 \mathrm{~b}$. The peak at $\sim 533.29 \mathrm{eV}$ matches well with the literature value for Sb- $3 d_{5 / 2}$ while the other peak appearing at higher energy, $\sim 533.88 \mathrm{eV}$, may arise due to the presence of physisorbed oxygen. The Sb- $3 d_{3 / 2}$ peak appeared at $\sim 543 \mathrm{eV}$. It may be observed that both the peaks for Sb$3 d_{5 / 2}$ and Sb- $3 d_{3 / 2}$ shifted by about $3 \cdot 7 \mathrm{eV}$ to higher energy than that indicated in the literature for the binding energy for native oxide or for major oxide species. The shift in O-1s peak does not match to confirm the possible formation of $\mathrm{Sb}_{2} \mathrm{O}_{4}$ or $\mathrm{Sb}_{2} \mathrm{O}_{5}$. Thus, possible oxidation of either $\mathrm{B}$ or $\mathrm{Sb}$ could be ruled out. The ratio of $\mathrm{B}: \mathrm{Sb}$ in the films could be quantified from the XPS spectra as $\sim 1: 1$ for all the films deposited under identical conditions as for the representative film. An additional peak at $\sim 202 \mathrm{eV}$ which was present in all the films deposited under identical condition could not be matched for any known element. We think that this may be associated with the compound, BSb, which could not be established on the basis of the existing knowledge on BSb compound. Thus, considering the results on the microstructural studies by XRD and TEM and the compositional studies by XPS, one may present the first report on the synthesis of $\mathrm{ZB}-\mathrm{BSb}$ in thin film form.

\subsection{Optical study}

The band gap of the BSb films were determined by standard technique of extrapolating the linear portion of the plot of $(\alpha h v)^{2}$ vs $h v$ which indicated $E_{g} \sim 0.52 \mathrm{eV}$. This value agrees well with the theoretical one, $\sim 0.53 \mathrm{eV}$, obtained from the band structure calculation using $0.512 \mathrm{~nm}$ as the equilibrium lattice constant. The optical constants (refractive index, $n$ and extinction coefficient, $k$ ) of the BSb films were determined. The refractive index was varied within $1 \cdot 65-2 \cdot 18$ with increasing energy of the incident photon. 
The plasma frequency, $\omega_{\mathrm{p}}$, was varied between $2 \cdot 370$ and $2.378 \times 10^{14} \mathrm{~s}^{-1}$ for all the films synthesized under identical conditions. The value of the carrier concentration, $N$, was found to be $\sim 2.813 \times 10^{18} \mathrm{~cm}^{-3}$.

\section{Conclusions}

Synthesis of BSb films by rapid thermal annealing of B/Sb multilayer film at $723 \mathrm{~K}$ for $3 \mathrm{~min}$ was demonstrated. The composition of the films was determined from XPS studies and $\mathrm{B} / \mathrm{Sb}$ ratio was found to be $\sim 1$. The band gap was an indirect one and the value was $\sim 0.51 \mathrm{eV}$. The electron diffraction pattern showed characteristic spots for planes
(111), (102) and (112). Two additional spots for reflection from (220) and (330) planes for ZB-BSb were also visible. The refractive index varied within $1 \cdot 65-2 \cdot 18$ with increasing energy of the incident photon. Plasma frequency $\left(\omega_{\mathrm{p}}\right)$ and the limiting value of the high frequency dielectric constant $\left(\varepsilon_{\infty}\right)$ were $2 \cdot 370-2.378 \times 10^{14} \mathrm{~s}^{-1}$ and $1 \cdot 88$, respectively. The effective mass was computed to be $\sim 0.0845 \mathrm{~m}_{\mathrm{e}}$.

\section{References}

Ferhat M et al 1998 J. Phys. Condens. Matter 107995

Kamashiro Y et al 2002 J. Cryst. Growth 1531237

Kamashiro Y et al 2004 J. Solid State Chem. 177533 